\title{
As borboletas Hesperiidae (Lepidoptera, Hesperioidea) do Distrito Federal, Brasil
}

\author{
Olaf Hermann Hendrik Mielke¹, Eduardo de Oliveira Emery² \& Carlos Eduardo Guimarães Pinheiro ${ }^{3}$
}

12Departamento de Zoologia, Setor de Ciências Biológicas, Universidade Federal do Paraná (UFPR), Caixa Postal 19020, Centro Politécnico,
81531-980 Curitiba-PR, Brasil. omhesp@ufpr.br
${ }^{2}$ Coordenação do Programa de Pesquisa em Biociências - COBIO/CNPq, SEPN QD. 509, Bloco A, Ed. Nazir, Sala 102. 70.750-501 Brasília,
Distrito Federal, Brasil. eduardoe@cnpq.br
${ }^{3}$ Departamento de Zoologia, Instituto de Biologia, Universidade de Brasília (UnB), 70910-900 Brasília, Distrito Federal, Brasil. cegp@unb.br

\begin{abstract}
The skippers (Lepidoptera, Hesperioidea, Hesperiidae) of the Distrito Federal, Brazil. An updated list containing 335 species of skippers (Hesperiidae) found in the Distrito Federal (central Brazil), including data obtained from the literature, from several entomological collections, and from collecting of the authors, is presented. Species with presumed distribution in the region are not included. It is presented too, a supplementary list with 32 endemic species occuring in the Cerrado vegetation, from which 27 were found in the Distrito Federal.
\end{abstract}

KEYWORDS. Central Brazil; cerrado; endemism; diversity; Neotropical.

\begin{abstract}
RESUMO. As borboletas Hesperiidae (Lepidoptera, Hesperioidea) do Distrito Federal, Brasil. Neste estudo é apresentada uma listagem atualizada com 335 espécies de borboletas Hesperiidae encontradas no Distrito Federal (Brasil central), incluindo dados da bibliografia, de várias coleções entomológicas e de coletas dos autores. Espécies de ocorrência apenas presumida não foram incluídas. Apresenta-se também uma lista suplementar com 32 espécies endêmicas do bioma Cerrado, das quais 27 foram encontradas no Distrito Federal.
\end{abstract}

PALAVRAS-CHAVE. Brasil Central; cerrado; endemismo; diversidade; Neotropical.

As listas de espécies de uma determinada área, feitas cuidadosamente por especialistas, constituem bases de dados confiáveis e perenes, servindo de referência para quaisquer estudos relacionados àquelas espécies. As listas contribuem para a divulgação da biodiversidade, conservação e monitoração ambiental, e auxiliam a identificação das espécies por profissionais e amadores, pois limitam a faixa de espécies que podem ocorrer em determinada região. Listar as espécies de uma região fornecendo os locais de suas ocorrências é fundamental para a elaboração de planos de conservação e métodos de avaliação ambiental. Brown \& Freitas (2000) utilizaram listas de espécies de borboletas de várias localidades da floresta Atlântica e Cerrado para o desenvolvimento de métodos de mensuração de biodiversidade e conservação. Observou-se, por exemplo, que a riqueza de espécies de Hesperiidae é um bom indicador para a riqueza total de fauna. De um modo geral, podemos assumir que uma grande riqueza de espécies de borboletas deve refletir uma grande riqueza de espécies de plantas, visto que suas larvas fitófagas tendem a ser especializadas (Diniz \& Morais 1995, 1997).

Neste estudo é apresentada uma lista de espécies de Hesperiidae que ocorrem no Distrito Federal (DF) (Tabela I) e são indicadas as espécies endêmicas do bioma Cerrado (Tabela II) que ocorrem no DF. Esta lista adicional de endêmicas constitui importante ponto de partida para o conhecimento do endemismo das borboletas do Cerrado, pois constatou-se que das 32 espécies de Hesperiidae endêmicas do Cerrado conhecidas até o momento, 27 ocorrem no DF. Assim, os objetivos deste estudo são: (1) apresentar uma lista das borboletas Hesperiidae do Distrito Federal; e (2) apresentar uma lista de borboletas Hesperiidae endêmicas do bioma Cerrado.

\section{MATERIALE MÉTODOS}

A lista de espécies de Hesperiidae do DF (Tabela I) apresentada a seguir foi baseada nas listagens de espécies de borboletas realizados por Brown \& Mielke (1967) nas florestas de Sobradinho e de galeria do Rio Sobradinho, Chapada da Contagem, região da Fercal, Brasília Country Clube, Jardim Zoológico de Brasília, Reserva Ecológica do Gama e Rio Maranhão; por Diniz \& Morais $(1995,1997)$ na Estação Ecológica da Universidade de Brasília (Fazenda Água Limpa); por Pinheiro \& Emery (2006) na Estação Ecológica do Jardim Botânico, Reserva Ecológica do IBGE, Estação Ecológica da Universidade de Brasília (Fazenda Água Limpa), Vila Telebrasília; no Parque Nacional de Brasília, na Estação Ecológica de Águas Emendadas, Reserva do Centro Olímpico (campus da Universidade de Brasília - UnB), Poço Azul, Reserva da Embrapa Cerrados (CPAC) Planaltina - DF, e em várias localidades na bacia do Rio Maranhão, além de dados obtidos nas coleções entomológicas da Universidade de Brasília (UnB), da coleção da Reserva Ecológica do Instituto Brasileiro de Geografia e Estatística (IBGE), Brasília, da Embrapa 
Cerrados (CPAC) Planaltina-DF, e da Coleção de Entomologia Pe. J. S. Moure, Departamento de Zoologia, Universidade Federal do Paraná, Curitiba, Paraná. Espécies com distribuições geográficas presumidas ou encontradas apenas em regiões vizinhas ao Distrito Federal não foram incluídas na presente lista. A classificação adotada segue Mielke (2005a-f).

A metodologia utilizada inclui coletas sistemáticas e ocasionais entre 9:00 e 17:00 horas, utilizando redes entomológicas ou atrativos de papel branco mascado, colocados sobre a vegetação, simulando fezes de aves. Os exemplares foram coletados obedecendo os requisitos de etiquetagem e procedimentos corretos de montagem e conservação, com acondicionamento em armários entomológicos com naftalina, em local com baixa umidade, controlada por aparelho desumidificador do ar.

As borboletas foram identificadas precisamente através de uma análise de exemplares montados em alfinetes entomológicos e freqüentemente foi necessário o exame da genitália. A identificação de espécies em campo é possível apenas para especialistas treinados, e ainda assim, somente possível para espécies maiores e vistosas. A maioria das espécies de Hesperiidae é de tamanho pequeno, com poucos caracteres conspícuos, ou coloração uniforme, com movimentos rápidos. Portanto, a identificação somente é possível após a coleta e montagem de exemplares em alfinete. Quando possível, é desejável a formação de séries de exemplares para cada região inventariada, pois isso possibilita a realização de estudos quantitativos e qualitativos. É desejável a coleta de exemplares de ambos os sexos, pois o dimorfismo sexual, comum à maioria das espécies de borboletas, torna a identificação das espécies uma tarefa menos difícil. As séries possibilitam a caracterização de variações intra-específicas, como fenótipos estacionais, ou seja, formas de inverno e verão, ou de ocorrência concomitante de diversos fenótipos geneticamente distintos, descontínuos dentro de uma mesma população. Espécies e subspécies ainda não descritas foram inventariadas neste estudo (Tabela I) e no estudo complementar da fauna de borboletas Papilionoidea do Distrito Federal, realizado por Emery et al. (2006).

A lista de espécies endêmicas (Tabela II) foi desenvolvida inicialmente a partir de dados de distribuição das espécies mencionadas na literatura (Evans 1951, 1952, 1953, 1955; Brown \& Mielke 1967; Mielke 1968) com posterior confirmação e ampliação de suas distribuições através da averiguação das coleções supracitadas. Neste estudo considera-se como espécies endêmicas do Cerrado aquelas que ocorrem no bioma Cerrado, em áreas de cerrado sensu stricto e suas variações, florestas mesofíticas, campos ou em florestas de galeria, dados verificados em etiqueta. Foram incluídos dados de distribuição geográfica nos estados do Brasil e a fitofisionomia onde foi coletada no bioma Cerrado. Para este estudo consideramos o bioma Cerrado exclusivamente brasileiro, com ocorrência nos estados do Ceará (CE), Maranhão (MA), Piauí (PI), Bahia (BA), Rondônia (RO), Tocantins (TO), Mato Grosso (MT), Mato Grosso do Sul (MS), Distrito Federal (DF), Goiás (GO), Minas Gerais (MG), São Paulo (SP), Paraná (PR), ocorrendo também em áreas disjuntas nos estados do Amapá (AP), Amazonas (AM), Pará (PA) e Roraima (RR) e Paraíba (PB) (Ratter et al. 1996; Lima-Verde 2004; Pereira \& Alves 2006; Von Linsingen et al. 2006). O Cerrado é bem caracterizado por Eiten (1992) e delimitado nos estados por Darrault \& Schlindwein (2005), Pereira \& Alves (2006) e Ratter et al. (1996). O Distrito Federal localiza-se na região central do bioma do Cerrado e possui áreas cobertas com diversas fitofisionomias, bem descritas em Eiten (1992).

\section{RESULTADOSEDISCUSSÃO}

Neste estudo é apresentada uma lista contendo 335 espécies de borboletas Hesperiidae (Lepidoptera, Hesperioidea) para a região do Distrito Federal (Tabela I), complementar ao estudo realizado por Emery et al. (2006), em que foram listadas 504 espécies de borboletas Papilionoidea. Assim, o número total de espécies de borboletas (Hesperioidea e Papilionoidea) no Distrito Federal chega a 839 espécies, número certamente provisório, pois com mais coletas em locais já estudados, ou mesmo novos, freqüentemente são encontradas espécies ainda não inventariadas. Este número expressivo representa aproximadamente $10,77 \%$ de toda a fauna de borboletas neotropical. Lamas (2004) menciona um total de 7.784 espécies de borboletas para esta região, destes 5.419 $(69,61 \%)$ são Papilionoidea e 2.365 (30,38\%) são Hesperioidea (Tabela III). No Distrito Federal 39,92\% de todas as espécies de borboletas são Hesperioidea, enquanto $60,07 \%$ são Papilionoidea. Se comparado ao total de 3.268 espécies de borboletas em território brasileiro, o número de 839 espécies de borboletas do DF, incluindo Papilionoidea e Hesperioidea, representa $25,67 \%$. Por outro lado, se for comparado o número total de espécies, as 839 espécies de borboletas do DF alcançam $39,95 \%$ do total aproximado de 2.100 espécies de borboletas da Floresta Atlântica, embora muitas borboletas do DF sejam de ocorrência amazônica, não ocorrendo na floresta atlântica (Brown \& Freitas 2000).

A maioria das espécies de Hesperioidea do DF tem distribuição muito ampla, ocorrendo em extensas áreas desde o Norte da América Central até o sul da América do Sul, em uma distribuição tipicamente Neotropical. Outras ocorrem em vários estados e países da América do Sul. Muitas espécies ocorrem em dois ou mais biomas, como no Cerrado e Floresta Amazônica, ou Cerrado e Floresta Atlântica, incluindo os campos de altitude (Evans 1951, 1952, 1953, 1955).

A lista preliminar de espécies endêmicas de Hesperiidae para o bioma Cerrado chega a 32 espécies, sendo que ocorrem duas subespécies de Sophista latifasciata, logo são 33 taxa. Sophista latifasciata latifasciata ocorre no Distrito Federal e nos estados de Goiás, Minas Gerais, Paraná e São Paulo, enquanto que Sophista latifasciata matto só é conhecida de Mato Grosso. A maioria, 27 espécies, ocorre no DF (Tabela II), no entanto, as cinco espécies ainda não encontradas possuem grande probabilidade de o serem com mais trabalho de campo. Interessante observar que mesmo as espécies endêmicas do Cerrado também apresentam uma distribuição ampla no bioma, 
Tabela I. Lista de borboletas Hesperiidae (Lepidoptera, Hesperioidea) do Distrito Federal, Brasília.

\begin{tabular}{|c|c|}
\hline & Hesperioidea - Hesperiidae \\
\hline & Pyrrhopyginae - Pyrrhopygini \\
\hline 1 & Elbella azeta giffordi Mielke, 1995 \\
\hline 2 & Elbella intersecta losca Evans, 1951 \\
\hline 3 & $\begin{array}{l}\text { Elbella luteizona (Mabille, 1877) - fenótipo sem manchas } \\
\text { alares amarelas }\end{array}$ \\
\hline 4 & Jemadia gnetus gnetus (Fabricius, 1781) \\
\hline 5 & Jemadia hewitsonii hewitsonii (Mabille, 1878) \\
\hline 6 & Jemadia menechmus (Mabille,1878) \\
\hline 7 & Microceris variicolor (Ménétriés, 1855) \\
\hline 8 & Mimoniades versicolor versicolor (Latreille, [1824]) \\
\hline 9 & Mysarbia sejanus stolli Mielke \& Casagrande, 2002 \\
\hline 10 & Mysoria barcastus barta Evans, 1951 \\
\hline 11 & Parelbella ahira extrema (Röber, 1925) \\
\hline 12 & Pyrrhopyge charybdis charybdis Westwood, 1852 \\
\hline 13 & Pyrrhopyge pelota Plötz, 1879 \\
\hline 14 & Pyrrhopyge sergius josephina Draudt, 1921 \\
\hline & Pyrginae - Eudamini \\
\hline 15 & Aguna albistria albistria (Plötz, 1880) \\
\hline 16 & Aguna asander asander (Hewitson, 1867) \\
\hline 17 & Aguna megacles megacles (Mabille, 1888) \\
\hline 18 & Aguna squamalba Austin \& Mielke, 1998 \\
\hline 19 & Astraptes alardus alardus (Stoll, 1790) \\
\hline 20 & Astraptes anaphus anaphus (Cramer, 1777) \\
\hline 21 & Astraptes enotrus (Stoll, 1781) \\
\hline 22 & Astraptes fulgerator fulgerator (Walch, 1775) \\
\hline 23 & Astraptes talus (Cramer, 1777) \\
\hline 24 & Augiades epimethea epimethea (Plötz, 1883) \\
\hline 25 & Autochton integrifascia (Mabille, 1891) \\
\hline 26 & Autochton itylus Hübner, 1823 \\
\hline 27 & Autochton longipennis (Plötz, 1882) \\
\hline 28 & Autochton neis (Geyer, 1832) \\
\hline 29 & Autochton zarex (Hübner, 1818) \\
\hline 30 & Bungalotis diophorus (Möschler, 1883) \\
\hline 31 & Bungalotis erythus (Cramer, 1775) \\
\hline 32 & Bungalotis midas (Cramer, 1775) \\
\hline 33 & Bungalotis quadratum barba Evans, 1952 \\
\hline 34 & Celaenorrhinus similis Hayward, 1933 \\
\hline 35 & Cephise malesedis Austin \& Mielke, 2000 \\
\hline 36 & Chioides catillus catillus (Cramer, 1779) \\
\hline 37 & Chrysoplectrum perna Evans, 1952 \\
\hline 38 & Chrysoplectrum pervivax (Hübner, [1819]) \\
\hline 39 & Codatractus aminias (Hewitson, 1867) \\
\hline 40 & Dyscophellus euribates euribates (Stoll, 1782) \\
\hline 41 & Dyscophellus nicephorus (Hewitson, 1876) \\
\hline 42 & Dyscophellus ramusis damias (Plötz, 1882) \\
\hline 43 & Entheus eunias Austin, Mielke \& Steinhauser, 1997 \\
\hline 44 & Epargyreus clavicornis clavicornis (Herrich-Schäffer, 1869) \\
\hline 45 & Epargyreus enispe enispe (Hewitson, 1867) \\
\hline 46 & Epargyreus exadeus exadeus (Cramer, 1779) \\
\hline 47 & Epargyreus socus socus Hübner, [1825] \\
\hline 48 & Nascus paulliniae (Sepp, [1842]) \\
\hline 49 & Nascus phocus (Cramer, 1777) \\
\hline 50 & Phanus australis L. Miller, 1965 \\
\hline 51 & Phanus marshalli (Kirby, 1880) \\
\hline 52 & Phocides pigmalion hewitsonius (Mabille, 1883) \\
\hline 53 & Phocides polybius phanias (Burmeister, 1880) \\
\hline 54 & Polygonus leo pallida Röber, 1925 \\
\hline 55 & Polythrix caunus (Herrich-Schäffer, 1869) \\
\hline 56 & Polythrix minvanes (Williams, 1926) \\
\hline 57 & Polythrix octomaculata (Sepp, [1844]) \\
\hline 58 & Proteides mercurius mercurius (Fabricius, 1787) \\
\hline 59 & Sarmientoia almeidai Mielke, 1967 \\
\hline 60 & Sarmientoia browni Mielke, 1967 \\
\hline 61 & Sarmientoia phaselis (Hewitson, 1867) \\
\hline 62 & Typhedanus undulatus (Hewitson, 1867) \\
\hline 63 & Udranomia kikkawai (Weeks, 1906) \\
\hline 64 & Udranomia orcinus (C. Felder \& R. Felder, 1867) \\
\hline 65 & Udranomia spitzi (Hayward, 1942) \\
\hline 66 & Urbanus albimargo takuta Evans, 1952 \\
\hline 67 & Urbanus chalco (Hübner, 1823) \\
\hline
\end{tabular}

Tabela I. Continuação.

\begin{tabular}{ll}
\hline 68 & Urbanus cindra Evans, 1952 \\
69 & Urbanus dorantes dorantes (Stoll, 1790) \\
70 & Urbanus doryssus doryssus (Swainson, 1831) \\
71 & Urbanus esmeraldus (Butler, 1877) \\
72 & Urbanus esta Evans, 1952 \\
73 & Urbanus evenus (Ménétriés, 1855) \\
74 & Urbanus procne (Plötz, 1880) \\
75 & Urbanus proteus proteus (Linnaeus, 1758) \\
76 & Urbanus simplicius (Stoll, 1790) \\
77 & Urbanus teleus (Hübner, 1821) \\
78 & Urbanus virescens (Mabille, 1877) \\
79 & Urbanus viterboana (Ehrmann, 1907)
\end{tabular}

\section{Pyrginae - Pyrgini}

80 Achlyodes busirus rioja Evans, 1953

81 Achlyodes mithridates thraso (Hübner, [1807])

82 Anastrus sempiternus simplicior (Möschler, 1877)

83 Anisochoria pedaliodina extincta Hayward, 1933

84 Anisochoria sublimbata Mabille, 1883

85 Anisochoria superior Mabille, 1898

86 Anisochoria vianna Evans, 1953

87 Antigonus erosus (Hübner, [1812])

88 Antigonus liborius liborius Plötz, 1884

89 Antigonus nearchus (Latreille, 1817)

90 Camptopleura auxo (Möschler, 1879)

91 Carrhenes canescens pallida Röber, 1925

92 Chiomara asychis autander (Mabille, 1891)

93 Chiomara basigutta (Plötz, 1884)

$94 \quad$ Chiomara mithrax (Möschler, 1879)

$95 \quad$ Clito bibulus (Riley, 1929)

$96 \quad$ Clito sompa Evans, 1953

97 Cogia abdul Hayward, 1947

98 Cogia calchas (Herrich-Schäffer, 1869)

$99 \quad$ Cogia cerradicola Mielke, 1967

$100 \quad$ Cogia grandis Riley, 1921

$101 \quad$ Cogia hassan evansi Bell, 1937

102 Cogia sp. n.

103 Cycloglypha polax Evans, 1953

104 Cycloglypha thrasibulus thrasibulus (Fabricius, 1793)

105 Ebrietas anacreon anacreon (Staudinger, 1876)

106 Eracon clinias (Mabille, 1878)

107 Erynnis (Erynnides) funeralis (Scudder \& Burgess, 1870)

108 Gesta austerus (Schaus, 1902)

109 Gesta gesta (Herrich-Schäffer, 1863)

110 Gesta heteropterus (Plötz, 1884)

111 Gindanes brebisson brebisson (Latreille, [1824])

112 Gindanes brontinus bronta Evans, 1953

113 Gorgythion begga begga (Prittwitz, 1868)

114 Gorgythion beggina escalophoides Evans, 1953

115 Gorgythion canda Evans, 1953

116 Gorgythion plautia (Möschler, 1877)

117 Grais stigmaticus stigmaticus (Mabille, 1883)

118 Helias phalaenoides palpalis (Latreille, [1824])

119 Heliopetes alana (Reakirt, 1868)

120 Heliopetes arsalte (Linnaeus, 1758)

121 Heliopetes macaira orbigera (Mabille, 1888)

122 Heliopetes omrina (Butler, 1870)

123 Heliopyrgus domicella willi (Plötz, 1884)

124 Iliana remus Bell, 1937

125 Milanion leucaspis (Mabille, 1878)

126 Mylon ander ander Evans, 1953

127 Mylon cristata Austin, 2000

128 Mylon maimon (Fabricius, 1775)

129 Mylon pelopidas (Fabricius, 1793)

130 Myrinia myris (Mabille, 1898)

131 Myrinia santa monka Evans, 1953

132 Nisoniades bipuncta (Schaus, 1902)

133 Nisoniades castolus (Hewitson, 1878)

134 Nisoniades macarius (Herrich-Schäffer, 1870)

135 Ouleus fridericus candangus Mielke, 1968

136 Pachyneuria inops (Mabille, 1877)

137 Pachyneuria duidae duidae (Bell, 1932)

138 Pellicia dimidiata zamia Plötz, 1882 
Tabela I. Continuação.

139 Pellicia hersilia Hayward, 1939

140 Pellicia ranta rancida Evans, 1953

141 Pellicia vecina vecina Schaus, 1902

142 Polyctor polyctor polyctor (Prittwitz, 1868)

143 Polyctor tensa Evans, 1953

144 Pyrgus orcus (Stoll, 1780)

145 Pyrgus orcynoides (Giacomelli, 1928)

146 Pythonides herennius lusorius Mabille, 1891

147 Pythonides jovianus fabricii Kirby, 1871

148 Pythonides lancea (Hewitson, 1868)

149 Pythonides limaea (Hewitson, 1868)

150 Quadrus cerialis (Stoll, 1782)

151 Quadrus fanda Evans, 1953

152 Quadrus jacobus (Plötz, 1884)

153 Quadrus u-lucida parabus Mielke, 1968

154 Sophista latifasciata latifasciata (Spitz, 1930)

155 Sostrata bifasciata adamas (Plötz, 1884)

156 Spathilepia clonius (Cramer, 1775)

157 Spioniades artemides (Stoll, 1782)

158 Staphylus melangon epicaste Mabille, 1903

159 Staphylus minor minor Schaus, 1902

160 Telemiades amphion misitheus Mabille, 1888

161 Telemiades corbulo ssp. n.

162 Telemiades laogonus laogonus (Hewitson, 1876)

163 Telemiades penidas (Hewitson, 1867)

164 Theagenes dichrous (Mabille, 1878)

165 Timochares trifasciata trifasciata (Hewitson, 1868)

166 Timochreon satyrus tampa Evans, 1953

167 Trina geometrina geometrina (C. Felder \& R. Felder, 1867)

168 Viola violella (Mabille, 1898)

169 Xenophanes tryxus (Stoll, 1780)

170 Xispia satyrus (Jörgensen, 1935)

171 Zera tetrastigma tetrastigma (Sepp, [1847])

172 Zopyrion evenor evenor Godman, 1901

173 Zopyrion reticulata Hayward, 1942

\section{Hesperiinae}

174 Aides duma duma Evans, 1955

175 Anatrytone perfida (Möschler, 1879)

176 Anthoptus epictetus (Fabricius, 1793)

177 Anthoptus insignis (Plötz, 1882)

178 Apaustus gracilis ssp. n.

179 Argon lota (Hewitson, 1877)

180 Arotis derasa derasa (Herrich-Schäffer, 1869)

181 Arotis kayei (Bell, 1932)

182 Artines acroleuca (Plötz, 1884)

183 Artines aepitus (Geyer, 1832)

184 Artines bipunctata Mielke, 1968

185 Artines sp. n.

186 Callimormus corades (C. Felder, [1863])

187 Callimormus juventus Scudder, 1872

188 Callimormus radiola pusillus Hayward, 1934

189 Callimormus saturnus (Herrich-Schäffer, 1869)

190 Calpodes ethlius (Stoll, 1782)

191 Cantha roraimae (Bell, 1932)

192 Carystoides basoches (Latreille, [1824])

193 Chalcone briquenydan briquenydan (Weeks, 1901)

194 Chalcone sp. n.

195 Chalcone zisa (Plötz, 1882)

196 Cobalopsis catocala (Herrich-Schäffer, 1869)

197 Cobalopsis cocalus (Hayward, 1939)

198 Cobalopsis miaba (Schaus, 1902)

199 Cobalopsis nero (Herrich-Schäffer, 1869)

200 Cobalus calvina (Hewitson, 1866)

201 Cobalus virbius hersilia (Plötz, 1882)

202 Conga chydaea (Butler, 1877)

203 Copaeodes castanea Mielke, 1969

204 Copaeodes jean favor Evans, 1955

205 Corticea corticea (Plötz, 1882)

206 Corticea lysias potex Evans, 1955

207 Corticea noctis (Plötz, 1882)

208 Corticea oblinita (Mabille, 1891)

209 Cumbre belli eberti Evans, 1955
Tabela I. Continuação.

$210 \quad$ Cyclosma altama (Schaus, 1902)

211 Cymaenes alumna (Butler, 1877)

212 Cymaenes cavalla Evans, 1955

213 Cymaenes chapa Mielke, 1968

214 Cymaenes chela Evans, 1955

215 Cymaenes gisca Evans, 1955

216 Cymaenes laureolus loxa Evans, 1955

217 Cymaenes laza Mielke, 1968

218 Cymaenes lepta (Hayward, 1939)

219 Cymaenes modestus (Hayward, 1943)

220 Cymaenes riba Mielke, 1968

221 Cymaenes tripunctus theogenis (Capronnier, 1874)

222 Cymaenes sp. n.

223 Cynea bistrigula (Herrich-Schäffer, 1869)

224 Cynea diluta (Herrich-Schäffer, 1869)

225 Cуnеa irma (Möschler, 1879)

226 Cynea popla Evans, 1955

227 Cynea robba nippa Evans, 1955

228 Decinea decinea decinea (Hewitson, 1876)

229 Decinea lucifer (Hübner, [1831])

230 Enosis uza uza (Hewitson, 1877)

231 Euphyes leptosema (Mabille, 1891)

232 Euphyes peneia (Godman, 1900)

233 Eutocus minor Mielke, 1967

234 Eutychide subcordata subcordata (Herrich-Schäffer, 1869)

235 Flaccilla aecas (Stoll, 1781)

236 Hylephila phyleus phyleus (Drury, 1773)

237 Justinia justinianus justinianus (Latreille, [1824])

238 Lento apta Evans, 1955

239 Lerema lineosa (Herrich-Schäffer, 1865)

240 Lerema veadeira Mielke, 1968

241 Lerodea erythrostictus (Prittwitz, 1868)

242 Lerodea eufala eufala (Edwards, 1869)

243 Lindra brasus brasus (Mielke, 1968)

244 Lucida lucia lucia (Capronnier, 1874)

245 Ludens petrovna (Schaus, 1902)

246 Lycas argentea (Hewitson, 1866)

247 Methionopsis ina (Plötz, 1882)

248 Metron oropa (Hewitson, 1877)

249 Metron zimra (Hewitson, 1887)

$250 \quad$ Mnaseas bicolor inca Bell, 1930

251 Mnasicles hicetaon Godman, 1901

252 Mnasitheus chrysophrys (Mabille, 1891)

253 Moeris striga striga (Geyer, 1832)

254 Morys geisa geisa (Möschler, 1879)

255 Morys sobra Mielke, 1968

256 Morys subgrisea subgrisea (Mabille, 1898)

257 Morys valerius valerius (Möschler, 1879)

258 Mucia scitula (Hayward, 1950)

259 Naevolus orius orius (Mabille, 1883)

260 Nastra chao (Mabille, 1898)

$261 \quad$ Nastra tanta Evans, 1955

262 Niconiades centralis Mielke, 1967

263 Niconiades nikko Evans, 1955

264 Niconiades xanthaphes Hübner, [1821]

265 Nyctelius nyctelius nyctelius (Latreille, [1824])

266 Onophas columbaria columbaria (Herrich-Schäffer, 1870)

267 Orses cynisca (Swainson, 1821)

268 Oxynthes corusca (Herrich-Schäffer, 1869)

269 Panoquina bola Bell, 1942

270 Panoquina chapada Evans, 1955

271 Panoquina fusina viola Evans, 1955

272 Panoquina hecebolus (Scudder, 1872)

273 Panoquina lucas lucas (Fabricius, 1793)

274 Panoquina ocola ocola (Edwards, 1863)

275 Panoquina trix Evans, 1955

276 Papias phainis Godman, 1900

277 Papias sp. n.

278 Papias subcostulata (Herrich-Schäffer, 1870)

279 Paratrytone argentea (Weeks, 1901)

280 Penicula advena advena (Draudt, 1923)

281 Perichares philetes adela (Hewitson, 1867)

282 Perichares seneca seneca (Latreille, [1824]) 
Tabela I. Continuação.

\begin{tabular}{ll}
\hline 283 & Phanes almoda (Hewitson, 1866) \\
284 & Phlebodes xanthobasis (Hayward, 1939) \\
285 & Polites vibex catilina (Plötz, 1886) \\
286 & Pompeius amblyspila (Mabille, 1898) \\
287 & Pompeius dares (Plötz, 1883) \\
288 & Pompeius pompeius (Latreille, [1824]) \\
289 & Pompeius postpuncta (Draudt, 1923) \\
290 & Pseudosarbia flavofasciata Skinner, 1921 \\
291 & Pyrrophygopsis socrates socrates (Ménétriés, 1885) \\
292 & Quinta cannae (Herrich-Schäeffer, 1869) \\
293 & Quinta locutia (Hewitson, 1876) \\
294 & Radiatus bradus Mielke, 1968 \\
295 & Remella remus (Fabricius, 1798) \\
296 & Saliana fusta Evans, 1955 \\
297 & Saliana longirostris (Sepp, [1840]) \\
298 & Saturnus metonidia (Schaus, 1902) \\
299 & Sodalia sodalis (Butler, 1877) \\
300 & Styriodes badius (Bell, 1930) \\
301 & Synale hylaspes (Stoll, 1781) \\
302 & Synale elana elana (Plötz, 1882) \\
303 & Synale metella (Plötz, 1882) \\
304 & Talides alternata Bell, 1941 \\
305 & Thespieus dalman (Latreille, [1824]) \\
306 & Tigasis fusca (Hayward, 1940) \\
307 & Vehilius gorta Evans, 1955 \\
308 & Vehilius inca (Scudder, 1872) \\
309 & Vehilius stictomenes stictomenes (Butler, 1877) \\
310 & Vettius artona (Hewitson, 1868) \\
\hline
\end{tabular}

Tabela I. Continuação.

\begin{tabular}{ll}
\hline 311 & Vettius arva Evans, 1955 \\
312 & Vettius fantasos (Cramer, 1780) \\
313 & Vettius lafrenaye pica (Herrich-Schäffer, 1869) \\
314 & Vettius lucretius (Latreille, [1824]) \\
315 & Vettius marcus marcus (Fabricius, 1787) \\
316 & Vettius richardi (Weeks, 1906) \\
317 & Vettius triangularis (Hübner, [1831]) \\
318 & Vidius felus Mielke, 1968 \\
319 & Vidius mictra Evans, 1955 \\
320 & Vidius nostra nostra Evans, 1955 \\
321 & Vidius similis Mielke, 1980 \\
322 & Vidius spitzi Mielke, 1967 \\
323 & Vidius tinta Evans, 1955 \\
324 & Vidius vidius (Mabille, 1891) \\
325 & Vinius exilis exilis (Plötz, 1883) \\
326 & Vinius letis (Plötz, 1883) \\
327 & Vinius tryhana istria Evans, 1955 \\
328 & Virga austrinus (Hayward, 1934) \\
329 & Wallengrenia otho sapuca Evans, 1955 \\
330 & Wallengrenia premnas (Wallengren, 1860) \\
331 & Xeniades chalestra chalestra (Hewitson, 1866) \\
332 & Xeniades orchamus orchamus (Cramer, 1777) \\
333 & Zariaspes mys (Hübner, [1808]) \\
& Heteropterinae \\
334 & Dalla diraspes (Hewitson, 1877) \\
& Dardarina jonesi Evans, 1955 \\
&
\end{tabular}

Tabela II. Espécies de borboletas Hesperiidae (Lepidoptera, Hesperioidea) endêmicas do Bioma Cerrado.

\begin{tabular}{|c|c|c|}
\hline & Pyrrhopyginae & Distribuição Geográfica \\
\hline 1 & Elbela intersecta losca Evans, 1951 & $\begin{array}{l}\text { Brasil (DF, GO). Floresta de galeria, mas pousa em areias úmidas nas beiras dos } \\
\text { riachos no cerrado. }\end{array}$ \\
\hline \multirow[t]{2}{*}{2} & Microceris variicolor (Ménétriés, 1855) & Brasil (DF, GO, MA, MG, MT, SP). Cerrado. \\
\hline & Pyrginae-Eudamini & \\
\hline 3 & Aguna camagura (Williams, 1926) & Brasil (GO, MT). Floresta de galeria e cerradão. \\
\hline 4 & Sarmientoia almeidai Mielke, 1967 & Brasil (DF, MS, MT, SP). Floresta de galeria e cerrado. Hábitos crepusculares. \\
\hline 5 & Sarmientoia browni Mielke, 1967 & Brasil (DF, GO, MG, MT, PI). Cerrado. Hábitos crepusculares. \\
\hline \multirow[t]{2}{*}{6} & Udranomia spitzii Hayward, 1942 & Brasil (BA, CE, DF, GO, MG, MT, PB, PR, SP). Cerrado. \\
\hline & Pyrginae-Pyrgini & \\
\hline 7 & Anisochoria vianna Evans, 1953 & Brasil (DF, GO, MG, MT). Cerrado e cerradão. \\
\hline 8 & Clito bibulus (Riley, 1929) & Brasil (DF, GO, MT, SP). Cerrado. \\
\hline 9 & Clito sompa Evans, 1953 & Brasil (BA, CE, DF, GO, MT, PA, SP). Cerrado e cerradão \\
\hline 10 & Cogia cerradicola (Mielke, 1967) & Brasil (BA, DF, GO, MT). Cerrado, normalmente em pequenos banhados. \\
\hline 11 & Cogia grandis Riley, 1921 & Brasil (BA, DF, GO, MG, MT, SP). Cerrado e cerradão. \\
\hline 12 & Cycloglypha polax Evans, 1953 & $\begin{array}{l}\text { Brasil (DF, GO, MG, MT, SP). Cerrado e cerradão, pousa em areias úmidas nas } \\
\text { beiras dos riachos. }\end{array}$ \\
\hline 13 & Milanion hemes memba Evans, 1953 & Brasil (GO, MG, MT). Floresta de galeria. \\
\hline 14 & Ouleus fridericus candangus Mielke, 1968 & Brasil (DF, GO). Floresta de galeria. \\
\hline 15 & Pythonides homer Evans, 1953 & Brasil ( MT). Floresta de galeria. \\
\hline 16 & Sophista latifasciata latifasciata Spitz, 1930 & Brasil (DF, GO, MG, PR, SP). Cerrado, cerradão e campo rupestre. \\
\hline 17 & Sophista latifasciata matto Evans, 1953 & Brasil (MT). Cerrado e cerradão. \\
\hline \multirow[t]{2}{*}{18} & Timochreon satyrus tampa Evans, 1953 & Brasil (DF, GO, MG, MT, DF). Floresta de galeria, cerrado e cerradão. \\
\hline & Hesperiinae & \\
\hline 19 & Artines bipunctata Mielke, 1968 & Brasil (DF, GO, MG). Floresta de galeria. \\
\hline 20 & Callimormus radiola pusillus Hayward, 1934 & Brasil (DF, GO, MT). Floresta de galeria. \\
\hline 21 & Cynea robba nippa Evans, 1955 & Brasil (DF, GO, MG, MT, PA). Floresta de galeria. \\
\hline 22 & Eutocus minor Mielke, 1967 & Brasil (DF, GO, MG). Cerrado. \\
\hline 23 & Lento apta Evans, 1953 & Brasil (DF, MT). Floresta de galeria. \\
\hline 24 & Lerema veadeira Mielke, 1968 & Brasil (BA, DF, GO, MG, PB, SP). Cerrado e campo rupestre. \\
\hline 25 & Metron schrottkyi tomba (Evans, 1955) & Brasil (MT). Floresta de galeria. \\
\hline 26 & Morys sobra Mielke, 1968 & Brasil (DF, MT). Cerrado. \\
\hline 27 & Nastra tanta Evans, 1955 & Brasil (DF, GO, MA, MG, MT, SP). Cerrado. \\
\hline 28 & Niconiades centralis Mielke, 1967 & Brasil (DF, GO). Floresta de galeria. \\
\hline 29 & Pompeius darina Evans, 1955 & Brasil (MT). Cerrado. \\
\hline 30 & Pseudosarbia flavofasciata Skinner, 1921 & Brasil (DF, MT). Cerrado. \\
\hline 31 & Vehilius gorta Evans, 1955 & Brasil (DF, GO, MG, MT). Cerrado. \\
\hline 32 & Vidius spitzi Mielke, 1967 & Brasil (AM, DF, MT). Cerrado. \\
\hline 33 & Vidius tinta Evans, 1955 & Brasil (DF, GO, MA, MT). Cerrado. \\
\hline
\end{tabular}


Tabela III. Comparação entre a diversidade de borboletas (Lepidoptera: Papilionoidea e Hesperioidea) da Região Neotropical e do Brasil com a do Distrito Federal.

\begin{tabular}{lcccl}
\hline Região & Papilionoidea & Hesperioidea & Total & Referências \\
\hline Neotropical & 5.419 & 2.365 & 7.784 & Lamas (2004) \\
Brasil & 2.103 & 1.165 & 3.268 & Brown (1996) \\
Distrito Federal & 504 & 335 & 839 & Emery et al. \\
& & & & $(2006)$ \\
\hline
\end{tabular}

seguindo o padrão da maioria das espécies de Hesperiidae.

Estas novas informações sobre alta diversidade de espécies de borboletas na região do DF reforçam a importância desta região para a conservação das espécies do Cerrado, principalmente em áreas de conservação como o Parque Nacional de Brasília, APA do Gama-Cabeça de Veado e Estação Ecológica das Águas Emendadas. Chama ainda atenção a necessidade de criação de outras áreas de conservação, principalmente na região da bacia do Rio Maranhão, norte do DF, com grande área de vegetação florestal em solo rico em calcário, evidentemente com grande riqueza de espécies e altamente ameaçada.

Agradecimentos. Os autores são gratos ao Dr. Amabílio José Aires de Camargo (EMBRAPA, Planaltina, Distrito Federal), Dra. Ivone Rezende Diniz (Universidade de Brasília, Brasília, Distrito Federal), ao pessoal da administração da Reserva Ecológica do IBGE pela permissão de estudo de exemplares sob suas curadorias e a dois revisores anônimos.

\section{REFERÊNCIAS}

Brown Jr., K. S. 1996. Diversity of Brazilian Lepidoptera: history of study, methods for measurement, and use as indicator for genetic, specific and system richness, pp. 221-153. In: Bicudo, C. E. De M. \& N. A. Menezes (eds). Biodiversity in Brazil. A first approach. São Paulo, Universidade de São Paulo, Instituto de Botânica. 326 pp.

Brown Jr., K. S. \& A. V. L. Freitas. 2000. Atlantic Forest butterflies: indicators for landscape conservation. Biotropica 32: 934-956.

Brown Jr., K. S. \& O. H. H. Mielke. 1967. Lepidoptera of the Central Brazil Plateau. II. Preliminary list of Rhopalocera (continued): Lycaenidae, Pieridae, Papilionidae, Hesperiidae. Journal of the Lepidopterists' Society 21: 145-168.

Darrault, R. O. \& C. Schlinwein 2005. Limited fruit production in Hancornia speciosa (Apocynaceae) and pollinisation by nocturnal and diurnal insects. Biotropica 37: 381-388.

Diniz, I. R. \& H. C. Morais 1995. Larvas de Lepidoptera e suas plantas hospedeiras em um cerrado de Brasília, Distrito Federal, Brasil. Revista Brasileira de Entomologia 39: 755-770.

Diniz, I. R. \& Morais, H. C. 1997. Lepidopteran caterpillar fauna of cerrado host plants. Biodiversity and Conservation 6: 817836.

Eiten, G. 1992. Natural Brazilian Vegetation Types and Their Causes. Anais da Academia brasileira de Ciências 64, Supl. 1: 36-65.

Emery, E. O.; K. S. Brown Jr. \& C. E. G. Pinheiro. 2006. As borboletas (Lepidoptera, Papilionoidea) do Distrito Federal, Brasil. Revista
Brasileira de Entomologia 50: 85-92.

Evans, W. H. 1951. A Catalogue of the American Hesperiidae Indicating the Classification and Nomenclature Adopted in the British Museum (Natural History). Part I. Pyrrhopyginae. British Museum, London. 92 pp.

Evans, W. H. 1952. A Catalogue of the American Hesperiidae Indicating the Classification and Nomenclature Adopted in the British Museum (Natural History). Part II. Pyrginae. Section I. British Museum, London. 178 pp.

Evans, W. H. 1953. A Catalogue of the American Hesperiidae Indicating the Classification and Nomenclature Adopted in the British Museum (Natural History). Part III. Pyrginae. Section II. British Museum, London. 246 pp.

Evans, W. H. 1955. A Catalogue of the American Hesperiidae Indicating the Classification and Nomenclature Adopted in the British Museum (Natural History). Part IV. Hesperiinae and Megathyminae. British Museum, London. 499 pp.

Lamas, G. 2004. Checklist: Part 4A. Hesperioidea - Papilionoidea. In: J. B. Heppner (ed.). Atlas of Neotropical Lepidoptera 5A. Association for Tropical Lepidoptera, Scientific Publishers. 439p.

Lima-Verde, L. W. 2004. Flora and autecology's aspects of a disjunction cerrado at Araripe plateau, Northeastern Brazil. Acta Botanica Brasilica 18: 759-770.

Mielke, O. H. H. 1968. Lepidoptera of the Central Brazil Plateau. New genera, species, and subspecies of Hesperiidae. Journal of the Lepidopterits' Society 22: 1-20.

Mielke, O. H. H. 2005a. Catalogue of the American Hesperioidea (Lepidoptera). Volume 1. Complementary and supplementary parts to the Checklist of the Neotropical Region. Hesperioidea: Hesperiidae: Pyrhopyginae. Volume 1, pp. XIII+1-125. Curitiba: Sociedade Brasileira de Zoologia.

Mielke, O. H. H. 2005b. Catalogue of the American Hesperioidea: Hesperiidae (Lepidoptera). Volume 2. Pyrginae 1: Eudamini, pp. 129-410 Curitiba: Sociedade Brasileira de Zoologia.

Mielke, O. H. H. 2005c. Catalogue of the American Hesperioidea: Hesperiidae (Lepidoptera). Volume 3. Pyrginae 2: Pyrgini, pp. 413-771. Curitiba: Sociedade Brasileira de Zoologia.

Mielke, O. H. H. 2005d. Catalogue of the American Hesperioidea: Hesperiidae (Lepidoptera). Volume 4. Hesperiinae 1: Adlerodea - Lychnuchus, pp. 775-1055. Curitiba: Sociedade Brasileira de Zoologia.

Mielke, O. H. H. 2005e. Catalogue of the American Hesperioidea: Hesperiidae (Lepidoptera). Volume 5. Hesperiinae 2: Megaleas - Zenis, pp. 1059-1381. Curitiba: Sociedade Brasileira de Zoologia.

Mielke, O. H. H. 2005f. Catalogue of the American Hesperioidea: Hesperiidae (Lepidoptera). Volume 6. Heteropterinae \& Megathyminae. Index, pp. 1387-1536 Curitiba: Sociedade Brasileira de Zoologia.

Pereira, M. do S. \& R. R. da N. Alves 2006. Composição florística de um remanescente de Mata Atlântica na Área de Preservação Ambiental Barra do Maranguape, Paraíba, Brasil. Revista de Biologia e Ciências da Terra 6: 357-365.

Pinheiro, C. E. G. \& E. O. Emery. 2006. As borboletas (Lepidoptera: Papilionoidea e Hesperioidea) da Área de Proteção Ambiental do Gama e Cabeça de Veado, Distrito Federal, Brasil. Biota Neotropica 6 h t t p ://ww w.biotaneotropica.org.br/v6 n $3 / \mathrm{pt} /$ abstract?inventory+bn01506032006. ISSN 1676-0603

Ratter, J. A.; S. Bridgewater, R. Atkinson \& J. F. Ribeiro. 1996. Analysis of the Floristic Composition of the Brazilian Cerrado Vegetation II: comparison of the Woody Vegetation of 98 areas. Edinburgh Journal of Botany 53: 153-180.

Von Linsingen, L.; J. S. Sonehara, A. Uhlmann \& A. Cervi. 2006. Composição florística do Parque Estadual do Cerrado de Jaguariaíva, Paraná, Brasil. Acta Biologica Paranaense 35: 197-232.

Recebido em 03/10/2007; aceito em 06/05/2008 